\title{
Clonal evolutionary particle swarm optimization for congestion management and compensation scheme in power system
}

\author{
N. Z. Mohd Ali ${ }^{1}$, I. Musirin' ${ }^{2}$, H. Mohamad ${ }^{3}$ \\ ${ }^{1}$ Faculty of Electric and Electronic Engineering, Universiti Malaysia Pahang (UMP), Malaysia \\ ${ }^{2,3}$ Faculty of Electric Engineering, Universiti Technology MARA, Malaysia
}

\begin{tabular}{l} 
Article Info \\
\hline Article history: \\
Received Jan 9, 2019 \\
Revised May 1, 2019 \\
Accepted May 25, 2019 \\
\hline Keywords: \\
Clonal evolutionary particle \\
swarm optimization (CEPSO) \\
Flexible AC transmission \\
system (FACTS) \\
Integrated multi-layer artificial \\
neural network (IMLANNs) \\
Static VAR compensator (SVC) \\
Thyristor control static \\
compensator (TCSC)
\end{tabular}

Corresponding Author:

\begin{abstract}
This paper presents computational intelligence-based technique for congestion management and compensation scheme in power systems. Firstly, a new model termed as Integrated Multilayer Artificial Neural Networks (IMLANNs) is developed to predict congested line and voltage stability index separately. Consequently, a new optimization technique termed as Clonal Evolutionary Particle Swarm Optimization (CEPSO) was developed. CEPSO is initially used to optimize the location and sizing of FACTS devices for compensation scheme. In this study, Static VAR Compensator (SVC) and Thyristor Control Static Compensator (TCSC) are the two chosen Flexible AC Transmission System (FACTS) devices used in this compensation scheme. Comparative studies have been conducted between the proposed CEPSO and traditional Particle Swarm Optimization (PSO). Results obtained by the developed IMLANNs demonstrated high accuracy with respect to the targeted output. Consequently, the proposed CEPSO implemented for single objective in single unit of SVC and TCSC has resulted superior results as compared to the traditional PSO in terms of achieving loss reduction and voltage profile improvement.
\end{abstract}

Copyright $(2019$ Institute of Advanced Engineering and Science. All rights reserved.

Nur Zahirah Mohd Ali,

Faculty of Electric and Electronic Engineering,

Universiti Malaysia Pahang, Malaysia.

Email: nurzahirah_mohdali@yahoo.com

\section{INTRODUCTION}

Congestion is an important issue in power system operation. Transmission congestion occurs when a power transmission system is unable to meet the needs of consumers due to higher demand. This gives an adverse effect on the electrical energy market where it cannot operate at a competitive level and may endanger the safety of the system [1]. Congestion management is a solution to address the issues of the operating system safely and consistently in an electric power system. The incidence of congestion can occur in several forms. Among these are caused by the failure of the transmission line, interference generator, changes in demand and clumsy dealing. The impact of congestion in a power system can lead to increased costs in certain areas and damage the device in the system [2].

Voltage stability phenomenon is a progressing issue in power system. It is one of the subsequent events which may occur due to congestion. It is normally measured using a stability index. C. Subramani in [3] proposed a Global Voltage Stability Index for voltage stability assessment. This index was employed with Artificial Neural Network to solve the voltage collapse problem. Since its introduction, ANN has become very popular, and applied to various decision making problem in numerous topics such as fault location [4-5], frequency, online ATC and real time power system estimation [6-8] voltage security evaluation [9], prediction of geothermal power plant system performance [10], and the rest of researchers was discussed about voltage stability monitoring [4, 11-13]. Today's scenario has faced of steady increase in demand of electricity. With the development of different FACTS devices, compensation effort has become 
another option for power system operators. Hence there is a need to study the optimum placement of FACTS devices. This is to avoid the over-compensation or under-compensation phenomena if no optimization process is involved. Various models for SVC, TCSC and PSS are conceivable and applied in different studies [14-22]. According to this issue, K. Vetrivel et al [23] suggests the implementation of Thyristor Controlled Series Capacitor in order to solve the congestion problem using Genetic Algorithm (GA) technique. In [24], the researcher highlighted a fuzzy technique to determine the optimal location of Thyristor Controlled Series Capacitor (TCSC). Therefore, the researcher used a Line Utilization Factor (LUF) and Real Power Performance Index (RPPI) factor to find out the level of congestion in a transmission line. Lastly, to observe the presence of TCSC, sensitivity parameters for the total system losses are derived as a function of the real power at the individual load points.

In this paper, Integrated Multi-Layer Artificial Neural Networks (IMLANNs) model has been developed for congested line prediction in choosing power system. Then, a new optimization technique termed as Clonal Evolutionary Particle Swarm Optimization (CEPSO) was developed in order to determine the optimal sizing of single unit of SVC and TCSC. The results highlight the advantage of new technique as compared to other technique with the objective function to minimize the transmission loss reduction and improvement of voltage profile in the system.

\section{RESEARCH METHOD}

\subsection{Integrated Multi-Layer Artificial Neural Networks (IMLANNs)}

Firstly, this research started with involves the development of a new model for determining the location of a FACTS device using artificial intelligence technique: namely Integrated Multi-Layer Artificial Neural Networks (IMLANNs) technique for a single objective function for predicting the Fast Voltage Stability Index $(F V S I)$ value and congested lines in power system. For the purpose of validation, the proposed technique is tested on two test systems, namely IEEE 30-Bus Reliability Test Systems (RTS). Before the IMLANNs model was developed, a case study was chosen to get an early result in power system load flow during normal and contingency condition. A pre-developed index, FVSI is used as a tool to identify the congested lines [25]. The experimental results of FVSI value is chosen as the targeted output in the IMLANNs model.

\subsubsection{Algorithm for the Proposed IMLANNs}

This section presents the whole procedures of the proposed IMLANNs model. The process of the proposed IMLANNs is explained below:

1. Choose the load bus. In IEEE 30-Bus RTS, there are 11 load buses that have been chosen for the testing process.

2. Read system data: IEEE 30-Bus RTS.

3. Set the loading condition. The system allows to run at the maximum permissible load.

4. Run load flow.

5. Calculate FVSI values for all lines and identify the congested lines. The equation of FVSI is show in (1).

$$
F V S I=\frac{4 Z_{l}^{2} Q_{r}}{V_{S}^{2} X_{l}}
$$

6. Build the raw data patterns for the ANN. If the number of patterns is not adequate, adjust the loading condition.

7. Cluster the training patterns and testing patterns.

8. Call the training data patterns.

9. Initialize the training parameters. On the stage of the training process, the numbers of nodes in each hidden layer, momentum rate, learning rate and other training parameters have been conducted by extensive experiments in order to determine the optimal value.

10. Develop the training configuration. The number of neurons in the first hidden layer, the number of neurons in the second hidden layer, the type of active of activation functions, number of iterative updates (epochs) and the mean square error goal can be varied in the process of determination optimal types or values.

11. Perform the training data patterns.

12. If the solution does not converge, vary the training parameters.

13. Save the IMLANNs model. 
14. Call the testing data patterns. Retrieve the saved IMLANNs model. The testing process is conducted consecutively after obtaining the best trained ANN model.

15. Perform testing process. Calculate the absolute error $\left(\mathrm{E}_{\mathrm{abs}}\right)$ as follows:

$$
E_{a b s}=\left|o_{c}-o_{p}\right|
$$

16. Where $O_{c}$ is the calculated output and $O_{p}$ is the predicted output. $n$ is the number of training patterns involved. The regression analysis is used to minimize the prediction error when fitting the predicted output to the targeted output.

17. If $R$ is not close to 1.00 , repeat step xii.

18. Tabulate the IMLANNs output and targeted output.

\subsection{Clonal Evolutionary Particle Swarm Optimization (CEPSO)}

Consequently, a hybrid optimization technique, namely Clonal Evolutionary Particle Swarm Optimization (CEPSO) is developed in order to determine the optimal sizing of single and multi-unit of FACTS device installation. The idea of hybrid CEPSO is developed based on the combination between Artificial Immune System (AIS), Evolutionary Programming (EP) and Particle Swarm Optimization (PSO) to improve the weakness experienced in the conventional PSO. This technique was built up to solve single objective problem (SOP) which considers minimizing the power loss and to improve the voltage profile.

\subsubsection{Algorithm of CEPSO Technique}

In this study, CEPSO is implemented in order to minimize the total losses in the distribution system; while monitoring voltage at all buses in the system to be within the acceptable limit. The step by step CEPSO algorithm for the proposed optimal sizing of FACTS devices is explained as follows:

1. Set the PSO parameter (Table 1).

Table 1. PSO Parameter

\begin{tabular}{cc}
\hline Paramater & Value \\
\hline Total number of particles, $\mathrm{N}_{\mathrm{p}}$ & $1,2,3$ \\
Maximum iteration, $\mathrm{K}_{\max }$ & 100 \\
Minimum weight, $\mathrm{W}_{\min }$ & 0.4 \\
Maximum weight, $\mathrm{W}_{\max }$ & 0.9 \\
Learning rate for individual ability, $\mathrm{C}_{1}$ & 2.05 \\
Social influence, $\mathrm{C}_{2}$ & 2.05 \\
Velocity constant factor, $\mathcal{E}$ & 1 \\
Minimum particle position, $\mathrm{X}_{\min }$ & 0 \\
Maximum particle position, $\mathrm{X}_{\max }$ & 1 \\
\hline
\end{tabular}

2. Set the parameters of the control variable to optimize as sizing of SVC and TCSC.

3. Set the loading condition for pre-optimization. Set the loss and voltage constraints, i.e. preLoss $\geq$ postLoss and preVoltage $\leq$ postVoltage, where preLoss and preVoltage are the loss and voltage profile values obtained by running load flow before any compensation process is conducted. This is important to certify that all generated initial population satisfy all the equality and inequality constraints.

4. Generate an initial population to consider the variable that should be optimized; the sizing of single or multi FACTS device. The random numbers $x$ is the control variables for the FACTS device $\left(x_{1}\right.$, $x_{2} \ldots x_{n}$ ) where $x_{n}$ is the sizing of FACTS devices to be installed into the system. The general matrix for initial population; $X_{s v c}$ and $X_{t c s c}$ is given by (3) and (4) respectively.

$$
\begin{aligned}
& X_{s v c}=\left[\begin{array}{lll}
x_{1} & x_{2} & x_{n}
\end{array}\right] \\
& X_{t c s c}=\left[\begin{array}{lll}
x_{1} & x_{2} & x_{n}
\end{array}\right]
\end{aligned}
$$

5. Initialize velocity and position.

6. Calculate Fitness 1. By considering the objective function, fitness is the particle in the population, which is evaluated and calculated by running the load flow.

7. The Clone process is a process to duplicate the parents. The mathematical equation is given in (5). Cloned Population, $\tau_{\text {nmk }}$ :- 


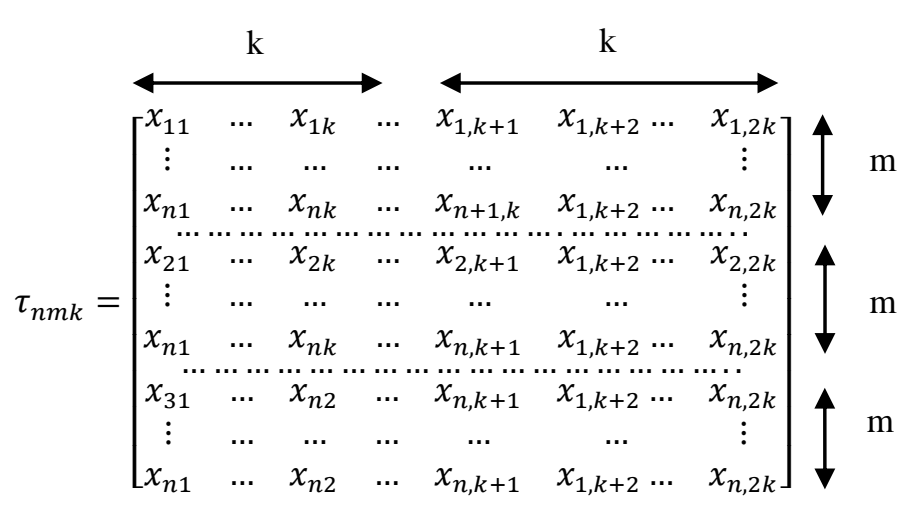

Matrix size of cloned population: $m n \times 2 k$

where:

$n=$ population number

$m=$ cloning number

$k=$ number of control variables

8. Calculate the new particles of position using mutation technique given in (6) and (7). Mutation is a process to produce offspring (children). $\beta$ is a scalar value, generated randomly to control the mutation process. $\beta$ controls the movement of candidates from the valley to hill within the chosen bell-curve. B can be pre-determined or randomized. For randomized $\beta$ value, this technique will become adaptive. This curve can be the Gaussian, Cauchy or Levy. The matrix sizing for the offspring is similar to that for the cloned population, $\tau_{n m k}$.

$$
\begin{aligned}
& x_{i+m, j}=x_{i, j}+N\left(0, \gamma^{2}\right) \\
& \gamma^{2}=\beta\left(x_{\text {jmax }}-x_{\text {jmin }}\right)\left(\frac{f_{i}}{f_{\text {max }}}\right)
\end{aligned}
$$

where:

$0 \leq \beta \leq 1=\operatorname{rand}(1,1)^{*} 1$

$\beta \quad=$ mutation scale

$f_{i} \quad=$ fitness for $i^{\text {th }}$ random number

$f_{\max } \quad=$ maximum fitness

$N \quad=$ Gaussian random variable

$x_{i, j} \quad=$ parents

$x_{\text {jmax }}=$ maximum random number for every variable

$x_{j m i n}=$ minimum random number for every variable

$x_{i+m, j}=$ mutated parents (offspring)

9. The combination is a process to group together the whole population and population after the cloned process in cascode form. It is can be conceptually represented as in (8), (9) and (10). From the parent population, $A_{1}$ and offspring population, $A_{2}$. Therefore, the combined population can be written as $C$.

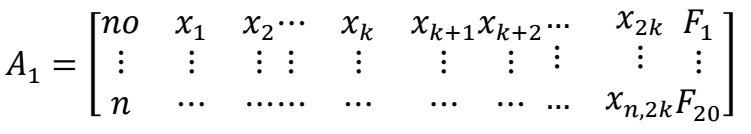

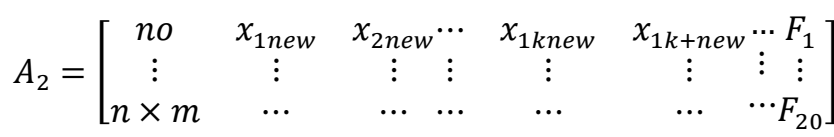

$$
\begin{aligned}
& C=\left[\frac{A_{1}}{A_{2}}\right]
\end{aligned}
$$

10. Tournament selection is a process to prescribe the candidates for the next iteration. If the cloning multiplier $m$ is 10 , therefore F1 and F2 will have 20x10=200 populations. Only 20 best members or individual is prescribed from this population for the next iteration. There are many techniques for the 
selection process such as pairwise comparison, elitism and a roulette wheel. Any suitable technique can be adopted for this purpose. But in this study, a pairwise comparison was used.

11. Find the $P_{\text {best_old }}$ and $G_{\text {best_old }}$ value and stored as $P_{\text {best_old }}=\min \left(P_{\text {best_fitness)_old }}\right.$ and $G_{\text {best_old }}=$

Pest_fitness_min_old.

12. Update the velocity and position of the particle.The condition to update velocity and position are described as follows:

$$
\begin{aligned}
& \text { Update velocity, If } \quad V_{i}^{k}>V_{\max } \\
& \text { set } V_{i}^{k}=V_{\max } \\
& \text { Else if } V_{i}^{k}<V_{\min } \\
& \text { set } V_{i}^{k}=V_{\min } \\
& \operatorname{set} x_{i}^{k}=x_{\max } \\
& \text { set } x_{i}^{k}=x_{\min }
\end{aligned}
$$

13. Calculate Fitness 2 and determine the $P_{\text {best_new }}$ and $G_{\text {best_new }}$ value. $P_{\text {best_new }}=\min \left(P_{\text {best_fitness }}\right)_{\text {new }}$ and $\mathrm{G}_{\text {best_new }}=\mathrm{P}_{\text {best Fitness_min_new. }}$

14. Perform convergence test. The convergence criterion is defined as the difference between Fitness and Fitness 2. Repeat steps (vii-ix) until stopping criterion, as such sufficiently excellent $G_{\text {best }}$ fitness or a maximum number of iteration is met.

15. End the CEPSO process.

\section{RESULTS AND ANALYSIS}

Firstly, loss minimization was chosen as the first objective function in this section. All the SVC locations were determined from the IMLANNs technique and the optimal sizing of single unit of SVC installation using the CEPSO technique in the IEEE 30-Bus RTS are tabulated in Table 2. Five load buses were chosen with their maximum reactive power loading in order to observe the installation of SVC to manage the congestion in power system. The participating load buses are buses 3, 4, 7, 10 and 12 . With maximum reactive loading, the congested line was identified based on the calculation of FVSI as the benchmark of congestion situation. From the congested line, the bus connected from bus to another bus can be known. The location of SVC was installed at the bus which experiencing congestion.

Table 2. Results of the single unit SVC installation using the CEPSO technique in IEEE 30- Bus RTS

\begin{tabular}{ccccccc}
\hline $\begin{array}{c}\text { Loaded } \\
\text { bus }\end{array}$ & $\begin{array}{c}\text {Q}_{\text {dmax }} \\
\text { (MVar) }\end{array}$ & $\begin{array}{c}\text { SVC Loc } \\
\text { (Bus) }\end{array}$ & $\begin{array}{c}\text { SVC Sizing } \\
\text { (MVar) }\end{array}$ & $\begin{array}{c}\text { Pre-opt } \\
\text { (MW) }\end{array}$ & $\begin{array}{c}\text { Post-opt } \\
\text { (MW) }\end{array}$ & (\%) $\Delta$ Loss \\
\hline 3 & 351.2 & 4 & 10 & 69.7726 & 65.1713 & 6.59 \\
4 & 391.6 & 12 & 10 & 49.4885 & 48.9081 & 1.17 \\
7 & 350.9 & 7 & 10 & 83.3981 & 76.9638 & 7.72 \\
10 & 182.0 & 11 & 10 & 30.1015 & 28.3162 & 5.93 \\
12 & 187.5 & 12 & 10 & 21.9468 & 21.5076 & 2.00 \\
\hline
\end{tabular}

$(\%) \Delta$ Loss $=\%$ of loss reduction

At $Q_{d 7}=350.9 \mathrm{MVar}$, Bus 7 has been determined as the SVC location while the optimal SVC sizing is $10 \mathrm{MVar}$ identified by using CEPSO. The percentage of minimum loss is $7.72 \%$, which is significantly high, compared to other load buses. For instance, at load Bus 3, the percentage of loss minimization show the second higher worth $6.59 \%$. The optimal SVC sizing is $10 \mathrm{MVar}$ while Bus 4 has been determined as the SVC location. From the observation, the overall result was shown the excellent reduction of loss minimization when the SVC was installed as a single unit in IEEE 30-Bus RTS.

TCSC was chosen as the second FACTS devices to manage congestion in power system. TCSC was installed on the line which apparently identified as congested line. The optimal location and sizing of TCSC was identified using CEPSO. Table 3 presents the results from the single unit TCSC installation using the CEPSO technique on the IEEE 30-Bus RTS. The table tabulates the optimal location and sizing of TCSC. The losses before and after CEPSO was implemented on the system are also shown. The percentage loss denotes the $\%$ of loss reduction with CEPSO implementation. On the overall, the power loss with TCSC 
installation has significantly reduced the loss for each loaded bus as shown in the table. The highest percentage of loss reduction of $3.14 \%$ when Line 15 was identified as the suitable TCSC location with its corresponding optimal sizing of 1.5531 p.u. At $Q_{d 10}=182.0 \mathrm{MVar}$, the optimal TCSC sizing is 0.6897 p.u., while the TCSC location is Line 13. At this condition, the percentage of loss reduction is $2.29 \%$. For optimal TCSC sizing at $Q_{d 12}$ of $187.5 \mathrm{MVar}$, is 0.3948 p.u. with the optimal location is Line 16 . The percentage of loss reduction is $1.79 \%$, reduction from $21.9468 \mathrm{MW}$ to $21.4118 \mathrm{MW}$.

Table 3. Results of single unit TCSC installation using CEPSO technique in IEEE 30-Bus RTS

\begin{tabular}{ccccccc}
\hline $\begin{array}{c}\text { Loaded } \\
\text { bus }\end{array}$ & $\begin{array}{c}\text { Qdmax } \\
\text { (MVar) }\end{array}$ & $\begin{array}{c}\text { TCSC Loc } \\
\text { (Line) }\end{array}$ & $\begin{array}{c}\text { TCSC Sizing } \\
\text { (p.u.) }\end{array}$ & $\begin{array}{c}\text { Pre-opt } \\
\text { (MW) }\end{array}$ & $\begin{array}{c}\text { Post-opt } \\
\text { (MW) }\end{array}$ & $\begin{array}{c}(\%) \\
\text { Loss }\end{array}$ \\
\hline 3 & 351.2 & 4 & 0.3674 & 69.7726 & 69.4052 & 0.53 \\
4 & 391.6 & 15 & 1.5531 & 49.4885 & 47.9354 & 3.14 \\
7 & 350.9 & 8 & 1.4213 & 83.3981 & 81.9768 & 1.70 \\
10 & 182.0 & 13 & 0.6897 & 30.1015 & 29.4118 & 2.29 \\
12 & 187.5 & 16 & 0.3948 & 21.9468 & 21.5520 & 1.79 \\
\hline
\end{tabular}

$(\%) \Delta$ Loss $=\%$ of loss reduction

In order to monitor the voltage profile improvement, the results of single unit SVC installation in IEEE 30-Bus RTS are illustrated as bar graph in Figure 1. In this figure, observation can be seen that the post voltage shows the voltage improvement as compared to the pre-voltage to all location of single unit SVC in the system. As mentioned in previous section, five SVC locations are chosen from the loaded buses. These locations are buses 4, 12, 7, 11 and 12 as seen on X-axis. For example, voltage after single unit of SVC is installed at Bus 4 has been improved to 1.0236p.u from its original value of $0.8264 \mathrm{p}$.u. The detail results of all single unit SVC installation are depicted in the figure.

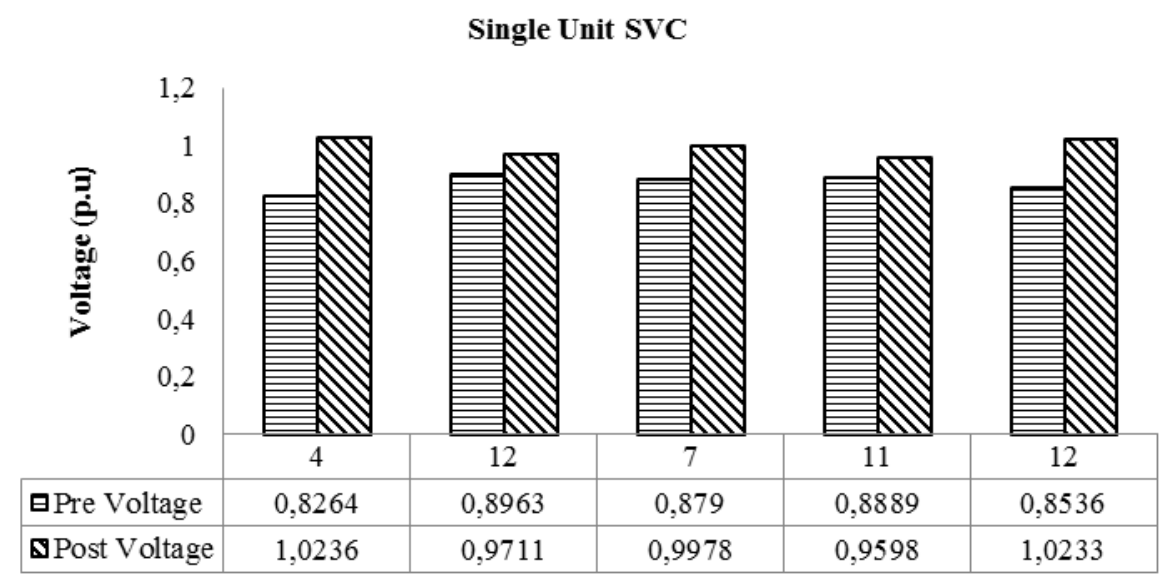

Figure 1. Results of voltage improvement when single unit SVC installation in IEEE 30-Bus RTS

Next study is the installation of the single unit of TCSC in the IEEE 30-Bus RTS. The results of voltage profile improvement when single unit of TCSC installation in IEEE 30-Bus RTS are presented in Figure 2. In this figure, observation can be seen that the post voltage shows the improvement as compared to the pre-voltage to all location of single unit TCSC in the system. As mentioned in the previous section, five TCSC locations are chosen from the loaded buses. These optimal locations are lines 4, 15, 8, 13 and 16 as seen on the x-axis. For example, voltage after single unit of TCSC is installed at Line 13 has improved to 0.9893p.u. from its original value of 0.9591p.u.. On the other hand, voltage before installation the single unit TCSC is increased when Line 15 is chosen as location of TCSC from 0.8632p.u. to 0.9411p.u.. The detail results of all single unit TCSC installation are depicted in the Figure 2. 


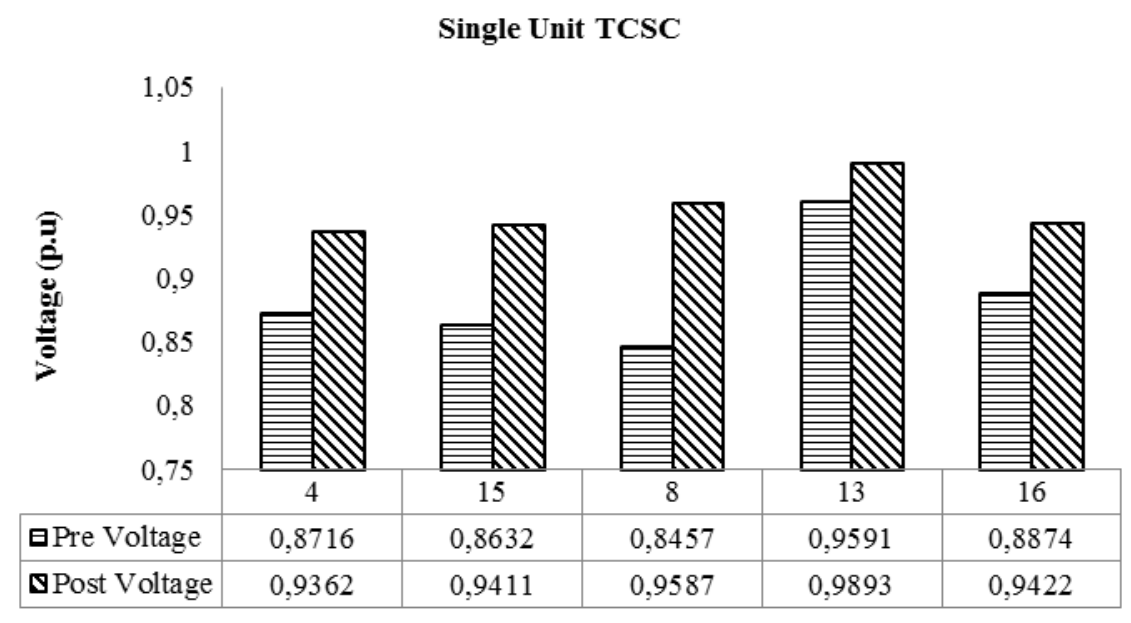

Figure 2. Results of voltage improvement when single unit TCSC installation in IEEE 30-Bus RTS

\section{CONCLUSION}

This paper has presented a new two technique as IMLANNs and CEPSO technique. IMLANNs technique was developed to predict the congested line in the system while CEPSO used to solve the single unit installation of SVC and TCSC for two independent objective functions. The first one is to minimize the loss; while the second objective is to improve the voltage profile. Both objectives have been successfully achieved through the implementation on IEEE 30-Bus RTS. The important effects to be highlighted in the results is the minimization of loss with the single unit of SVC and TCSC installation into the system. From the results, it is observed that the installation of single unit of SVC and TCSC with the optimal locations and sizing can help the power system operator for their future planning.

\section{REFERENCES}

[1] J. Hazra, et al., "Congestion Management Using Multiobjective Particle Swarm Optimization," IEEE Transactions on Power Systems, vol. 22, pp. 1726-1734, 2007.

[2] S. Parnandi, et al., "Power market analysis tool for congestion management," Proceedings International Conference on Electrical Engineering Design and Technologies (ICEEDT), Nov 2007.

[3] C. Subramani, et al., "Artificial neural network based voltage stability analysis in power system," 2016 International Conference on Circuit, Power and Computing Technologies (ICCPCT), pp. 1-4, 2016.

[4] S. Singh and D. N. Vishwakarma, "ANN and wavelet entropy based approach for fault location in series compensated lines," 2016 International Conference on Microelectronics, Computing and Communications (MicroCom), pp. 1-6, 2016.

[5] L. Tekli, et al., "Artificial neural network approach for locating faults in power transmission system," EUROCON, 2013 IEEE, pp. 1425-1430, 2013.

[6] K. D. Kartik, et al., "Frequency estimation of power system using CMAC Artificial Neural Network," 2016 International Conference on Circuit, Power and Computing Technologies (ICCPCT), pp. 1-5, 2016.

[7] V. A. I. Selvi, et al., "Artificial neural network approach for on-line ATC estimation in deregulated power system," Power Signals Control and Computations (EPSCICON), 2014 International Conference on, pp. 1-5, 2014.

[8] H. Mosbah and M. El-Hawary, "Multilayer artificial neural networks for real time power system state estimation," Electrical Power and Energy Conference (EPEC), 2015 IEEE, pp. 344-351, 2015.

[9] A. Zhukov, et al., "A hybrid artificial neural network for voltage security evaluation in a power system," Energy (IYCE), 2015 5th International Youth Conference on, pp. 1-8, 2015.

[10] D. Ruliandi, "Geothermal power plant system performance prediction using artificial neural networks," IEEE Conference on Technologies for Sustainability, pp. 216-223, 2015.

[11] S. Messalti, et al., "Artificial neural networks controller for power system voltage improvement," Renewable Energy Congress (IREC), 2015 6th International, pp. 1-6, 2015.

[12] C. M. d. Cruz, et al., "Application of Paraconsistent Artificial Neural Network in Statistical Process Control acting on voltage level monitoring in Electrical Power Systems," Intelligent System Application to Power Systems (ISAP), 2015 18th International Conference on, pp. 1-6, 2015.

[13] A. R. Bahmaniar and A. Karami, "Power system voltage stability monitoring using artificial neural networks with a reduced set of inputs," International Journal of Electrical Power \& Energy Systems, vol. 58, pp. 246-256, 2014.

[14] X. Wang, et al., "Optimal allocation of transmission rights in systems with FACTS devices," Generation, Transmission and Distribution, IEE Proceedings, vol. 149, pp. 359-366, 2002. 
[15] S. Shivashankar, "Optimal location of TCSC in transmission lines using contingency severity index and performance index methods for single contingency using PSO," Power, Energy and Control (ICPEC), 2013 International Conference on, pp. 135-138, 2013.

[16] Y. del Valle, et al., "Optimal STATCOM Sizing and Placement Using Particle Swarn Optimization,” Transmission \& Distribution Conference and Exposition: Latin America, 2006. TDC '06. IEEE/PES, pp. 1-6, 2006.

[17] B. Mahdad and K. Srairi, "Security optimal power flow considering loading margin stability using hybrid FFA-PS assisted with brainstorming rules," Applied Soft Computing, vol. 35, pp. 291-309, 2015.

[18] T. Kamel, et al., "Optimal number, location and parameter setting of multiple TCSCs for security and system loadability enhancement," Systems, Signals \& Devices (SSD), 2013 10th International Multi-Conference on, pp. 16, 2013.

[19] B. Bhattacharyya and V. K. Gupta, "Fuzzy based evolutionary algorithm for reactive power optimization with FACTS devices," International Journal of Electrical Power \& Energy Systems, vol. 61, pp. 39-47, 2014.

[20] L. Srivastava, et al., "Optimal location and size of TCSC for voltage stability enhancement using PSO-TVAC," Power and Energy Systems Conference: Towards Sustainable Energy, pp. 1-6, 2014.

[21] S. Dixit, et al., "An Overview of Placement of TCSC for Enhancement of Power System Stability," pp. 1184-1187, 2014.

[22] H. Shayeghi, et al., "Simultaneous optimal placement and parameter-tuning of SVC, TCSC and PSS using HoneyBee Mating Optimization," Power and Energy Engineering Conference (APPEEC), 2013 IEEE PES Asia-Pacific, pp. 1-5, 2013.

[23] K. Vetrivel, et al., "Optimal placement and control of TCSC for transmission congestion management," 2014 International Conference on Computation of Power, Energy, Information and Communication (ICCPEIC), pp. 281288, 2014.

[24] S. S. P. Smt. Ushasurendra, "Congestion management in deregulated power sector using fuzzy based optimal location technique for series flexible alternative current transmission system (FACTS) device," Journal of Electrical and Electronics Engineering Research, vol. 4, pp. 12-20, 2012.

[25] I. Musirin, "New techniques for voltage stability assessment and improvement in power system," Phd Thesis Phd Thesis, Electrical Engineering, Universiti Teknologi MARA Malaysia, 2002. 